\title{
GOALKEEPER GAME: A NEW ASSESSMENT INSTRUMENT IN NEUROLOGY SHOWED HIGHER PREDICTIVE POWER THAN MOCA FOR GAIT PERFORMANCE IN PEOPLE WITH PARKINSON'S DISEASE
}

Rafael B. Stern ${ }^{1}$, Matheus d'Alencar ${ }^{2}$, Yanina L. Uscapi ${ }^{3}$, Marco D. Gubitoso $^{4}$, Antonio C. Roque ${ }^{5}$, André F. Helene ${ }^{3}$, and Maria Elisa P. Piemonte $^{2}$

${ }^{1}$ Department of Statistics, UFSCar, São Carlos, SP, Brazil

${ }^{2}$ Departament of Physical Therapy, Speech Therapy and

Occupational Therapy, USP, São Paulo, SP, Brazil

${ }^{3}$ Department of Physiology, USP, São Paulo, SP, Brazil

${ }^{4}$ Department of Computer Science, USP, São Paulo, SP, Brazil

${ }^{5}$ Department of Physics, USP, Ribeirão Preto, SP, Brazil

August 25, 2018

Objective: To investigate the use of the Goalkeeper Game (GG) to assess gait automaticity decline under dual task conditions in people with Parkinson's disease (PPD) and compare its predictive power with the one of the MoCA test.

Materials and Methods: 74 PPD (H\&Y stages: 23 in stage 1; 31 in stage 2; 20 in stage 3), without dementia (MoCA cut-off 23), tested in ON period with dopaminergic medication were submitted to single individual cognitive/motor evaluation sessions. The tests applied were: MoCA, GG, dynamic gait index (DGI) task and timed up and go test (TUG) under single 
and dual-task (DT) conditions. GG test resulted in 9 measures extracted via a statistical model. The predictive power of the GG measures and the MoCA score with respect to gait performance, as assessed by DGI and DT-TUG, were compared.

Results: The predictive models based on GG measures and MoCA score obtained, respectively, sensitivities of $65 \%$ and $56 \%$ for DGI scores and $59 \%$ and $57 \%$ for DT-TUG cost at a $50 \%$ specificity. GG application proved to be feasible and aroused more motivation in PPDs than MoCa.

Conclusion: GG, a friendly and ludic game, was able to reach a good power of gait performance prediction in people at initial and intermediate stages of PD evolution.

\section{Introduction}

Parkinson's disease (PD) daily living independence decrease and, consequently, the reduction in Health-Related Quality of Life (HRQoL) [40], are directly associated to deficiency in automaticity. One of the most devastating deficiencies involves gait impairments, product of inability of the nervous system to successfully coordinate movement, driving patients to a gradual increase in cortical demand to execute basic motor operations via attentional processes [6, 64, 13].

Although gait description typically involves an implicit ability that can be automatically performed, during tasks involving complex motor and perceptual integration, gait performance can become dependent on both automatic (implicit) and attentional (explicit) guided processes $[65,67,2]$. As in people with PD (PPD), even the gait parameters controlled by automatic processes in healthy individuals become dependent on attentional control [64, 12, 37], simple tasks become complex actions and complex ones, as walking over obstacles [53, 19, 1], avoiding obstacles [43], or managing unexpected targets and obstacles [8], can be impracticable. Moreover, assuming that each individual has a certain maximum attentional reserve capacity, when more attentional resources are needed to compensate for the deficits in automaticity, less of these resources remain to be used for other simultaneous tasks. The impact of this tradeoff can, sometimes, hide actual gait deficiency but is always noticeable in the impact of a concurrent cognitive task in PPD [50].

In fact, dual task (DT) gait paradigm, e.g., walking while performing a second task, is commonly applied to investigate gait automaticity in PD. DT gait paradigm allows the assessment of effects of attention division, permitting the detection of gait deficits that under single task (ST) (walking with no other assigned task) condition could be undetectable [7]. Gait evaluation under DT has been stated as a reliable measure for clinical studies in PD [55]. The change in gait performance under DT conditions relative to the ST condition is called dual-task cost (DTC) [13]. DTC has been largely demonstrated to be a reliable measure of impaired DT gait performance in PD [65, 66, 44, 47, 3, 25, 59].

The association between cognitive and motor disability performances provides a basis for understanding the complex role of cognition in Parkinsonian gait. The impaired 
ability of PPD to adapt the stepping and walking behavior towards targets and obstacles has significant correlations with the Montreal Cognitive Assessment (MoCA) score, which may reflect less effective behavioral responses due to attentional control deficits and/or impaired cognitive function [45]. In clinical routine, MoCA test is widely used to evaluate the cognitive status in PPD [11, 22, 29, 42, 51], being able to detect alterations even in early stages of the disease [33]. The MoCA test is a useful screening tool for global cognitive and executive functions in PD [27]. However, DT costs combined with clinical confounders were able to explain only about $30 \%$ of the MoCA score variance [20].

New tools may provide alternative and sensitive methods of early and non-invasive automaticity decline screening in PD. Identification of early automaticity impairment could provide a critical opportunity for early intervention before gait changes with major impact on independence in daily living activity, fall risk, and HRQoL. The Goalkeeper Game (GG) was introduced in de Castro [15] as a tool to investigate the conjecture that the brain does statistical model selection. GG is a videogame with internet, desktop and mobile device versions (http://game.numec.prp.usp.br) in which the player, taking the role of a goalkeeper in a soccer penalty shootout, guesses the position in the goal that the ball will hit (left side, right side or center). The game consists in a sequence of penalty kicks in which the ball positions can be generated either deterministically or randomly according to a strategy described by a tree and unknown to the player. The strategy is fixed for each phase and as the player (the goalkeeper) succeeds in guessing enough hits, which depends on the strategy tree, the phase terminates and a new one starts with a more complex tree. As the game evolves, the expectation is that for large numbers of trials in each phase the player is able to make sense of the strategy and obtain a high-scoring performance. Currently, the GG is being used by the Research, Innovation and Dissemination Center for Neuromathematics (http://neuromat.numec.prp.usp.br/) as an assessment tool in its basic and applied neuroscience researches.

GG allows for massive data collection, and it is expected that statistical analyses of the players' hit rates can discriminate between explicit and implicit learning (EL and IL) impairment processes behind the players' decision-making models. Assuming that IL system is the groundwork to build automaticity, it is plausible to suppose that GG is able to predict automaticity gait decline in people with PD. Then, the purpose of this study was to investigate the predictive power of GG in comparison with MoCA for gait automaticity impairments in PPD.

\section{MATERIALS AND METHODS}

\subsection{Participants}

A convenient sample of seventy-four PPD recruited from the Laboratory of Motor Learning participated in this study. Inclusion criteria involved were individuals with (1) idiopathic Parkinson's disease as diagnosed by an experienced specialist in movement disorders, following the UK Brain Bank criteria [31], taking antiparkinsonian medications, (2) in 1-3 disease stage according to Hoehn and Yahr scale [28], (3) able to ambulate independently, (4) no signals of dementia (as determined by MoCA - cut-off 25) and/or 


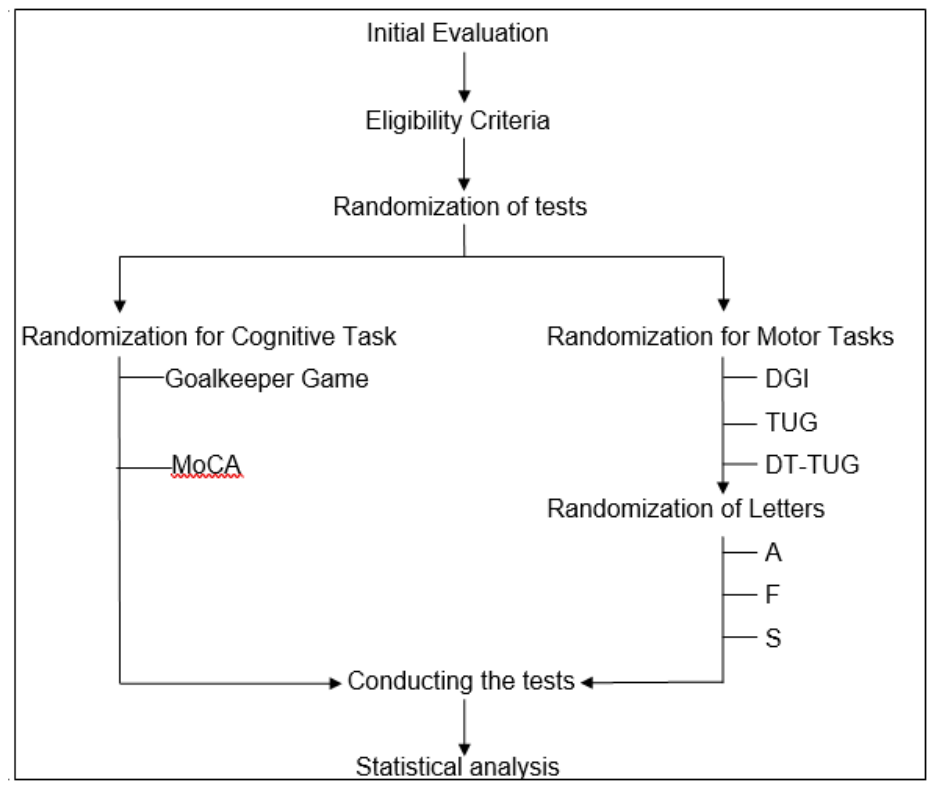

Figure 1: Study design flowchart.

major depression (as determined by Geriatric depression scale - cut-off 6). Subjects were excluded if they had clinically significant musculo-skeletal, cardio-vascular or respiratory disease, other neurological disease, or uncorrected visual/auditive disturbances.

\subsection{Design and procedures}

This study was approved by a Local Ethical Committee (CAAE 67388816.2.0000.0065) and conducted in accordance with the Helsinki Declaration. A written informed consent was signed for each participant before the study begun.

Based on a cross-sectional design, participants completed motor and cognitive evaluation in a single section. Evaluation order was randomized by sortition (Figure 1). Individual evaluation was conducted by a nurse and a physiotherapist specialized in movement disorders. All participants with PD were tested 40 to 120 minutes after their L-dopa dose (ON period).

\subsection{Cognitive evaluation}

Participants performed cognitive evaluation ( $\mathrm{GG}$ and MoCA), seated comfortably in front of a desk where they could place elbows and forearms.

\subsubsection{GG evaluation}

GG was presented in a 23" monitor (height $=29 \mathrm{~cm}$, width $=51 \mathrm{~cm}$ ) positioned $60 \mathrm{~cm}$ in front of the participants. After initial explanation about the game's rules, participants are asked to assume the goalkeeper role during the penalty shootout, pressing the selected 


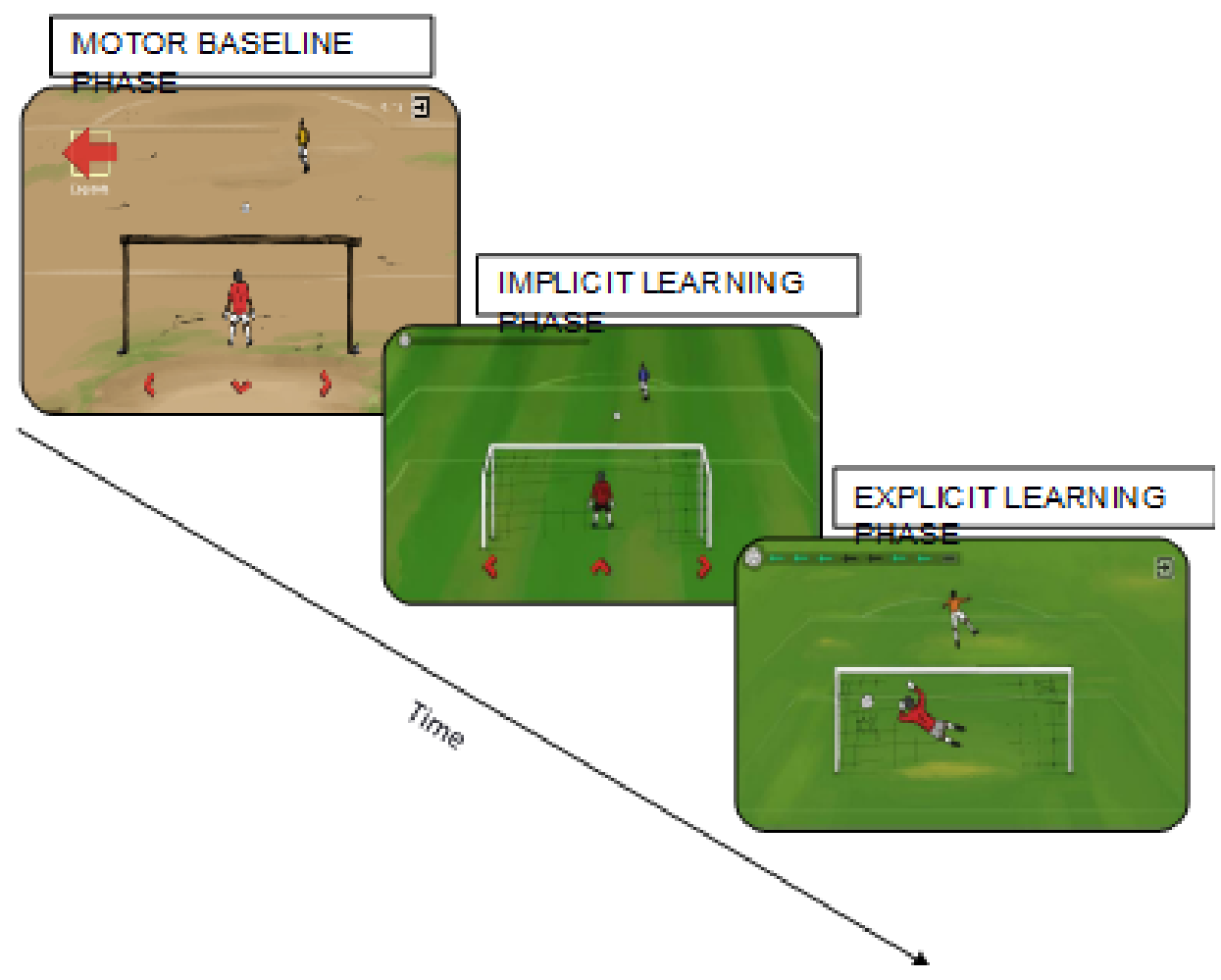

Figure 2: Goalkeeper Game where the participant was instructed to control the movements of a goalkeeper in time for a penalty, needing to guess the direction in which the opponent kicked the ball (left, right or center). At each level, the opponent adopted a new strategy based on implicit or explicit learning.

key among three possibilities ( $\triangleleft, \mathbf{\nabla}$ or $\boldsymbol{\text { ) }}$ ) which controls the direction of the goalkeeper's movement to defend the penalty (left, center or right).

The GG version used in this study is a simplified one with only deterministic penalty sequences. It has 3 phases: (1) MOTOR BASELINE PHASE, in which visual cues were offered to participants to guide the correct direction (5 trials); (2) IL PHASE, when no cues were offered and the sequences were provided by a deterministic model (20 trials); and (3) EL PHASE, in which participants were asked to memorize the correct sequence before playing the game (5 trials) (Figure 2).

After every trial, participants received visual and auditory feedback indicating successful or unsuccessful attempt. All participants had to complete the three phases regardless of their performance.

\subsubsection{MoCA evaluation}

The MoCA consists of 12 individual tasks that are scored and summed with a 6-item orientation screening and an educational correction (i.e., one point added for individuals with 12 years of education or less) to generate a total score representing global cognitive 
functioning. The total score is 30 points among seven cognitive domains, which reflect similar constructs as those assembled from a more comprehensive neuropsychological battery [60]. MoCA was administered with paper-and-pencil by a nurse with appropriate training. Participants received no feedback and completed the assessment regardless of their performance.

\subsection{Motor evaluation}

The motor evaluations were performed in a large room with adequate illumination and floor surface by a trained physiotherapist.

\subsubsection{Dynamic Gait Index (DGI)}

This test assesses gait performance during 8 gait-related activities. These include quality of walking speed change, going around and over obstacles and stair walking as well as number of steps required for a pivot turn. Performance is scored from 0 to 24 indicating, respectively, lowest and highest functioning level [30]. DGI is highly recommended as a test to assess gait performance and has demonstrated good feasibility, test-retest and interrater reliability in PD. Furthermore, it is considerably useful as a supportive test for identifying the fall risk of people with PD [32]. After the initial explanation about the test, participants were asked to walk in habitual speed following examiner's instructions.

\subsubsection{Dual-task Timed up to go test (DT-TUG)}

TUG is a widely used test capable of providing valuable information on balance and mobility in PD [41, 49,35]. This test measures the time in seconds that a patient takes to get up from a chair, walk $3 \mathrm{~m}$, make an $180^{\circ}$ turn and go back to the original sitting position. Although walking is a large component of the test, its execution demands more than walking and it is unique in its serial integration of several mobility tasks. TUG has been considered an instrument with reliability and construct validity in PD [41, 4, 14] and is strongly recommended to assess gait performance and mobility in PD [5].

DT-TUG, i.e., TUG performed concomitantly with another task, has been used to increase TUG sensibility. DT-TUG is more sensitive than single-task TUG in identifying frail and prefrail individuals [36, 58], impairment in focused attention [10] and fallers [46] in elderly and PPD [56]. The concurrent cognitive task used in DT-TUG in this study consisted of speaking as many words as possible starting with a specific letter ( $\mathrm{F}, \mathrm{S}$ or A) presented at the beginning of test. After initial explanation, participants were asked to stand up from a chair, walk $3 \mathrm{~m}$ at normal speed, turn around, and come back to sit in the chair. Concomitantly, they had to complete a cognitive task (DT-TUG) or not (TUG). The tests order was randomized. 


\section{Statistical analysis}

The GG variables cannot be compared directly to other clinical variables. For instance, for each stage and patient, the GG consists of a sequence of failures and successes in the patient's predictions for that stage. Since each particular prediction carries little information, it is weakly correlated to the patient's clinical variables.

In order to overcome this problem, we built a model which extracts each patient's overall performance in the GG. This model is a generalization of logistic regression [17][p.119]. Specifically, for each time iteration, $t$, patient, $p$, and stage of GG, $s$, we define $X_{t, p, s}$ as the indicator that $p$ made the correct prediction at iteration $t$ of stage $s$ of the GG. The distribution of $X_{t, p, s}$ is given by

$$
\begin{aligned}
\lambda_{t, p, s} & =(t-1) \beta_{p, s}-\log \left(3 \gamma_{p, s}-1\right) \\
\mathbb{P}\left(X_{t, p, s}=1\right) & =\gamma_{p, s} \frac{\exp \left(\lambda_{t, p, s}\right)}{1+\exp \left(\lambda_{t, p, s}\right)}
\end{aligned}
$$

The model in eq. 1 admits an intuitive interpretation. First, it is chosen so that the probability that a patient makes a correct prediction at the first iteration of each stage is $\frac{1}{3}$. This is reasonable since, at that point, the patient has no information and there are 3 options. Also, the parameter $\gamma_{p, s}$ represents the limit of patient $p$ 's learning at stage $s$. That is, $\gamma_{p, s}$ is the probability that $p$ makes a correct prediction at $s$ after playing that stage for a large number of iterations. Finally, $\beta_{p, s}$ is the rate of learning of patient $p$ at stage $s$. That is, $\beta_{p, s}$ determines how many iterations $p$ requires at stage $s$ so that his probability of making a correct prediction is close to $\gamma_{p, s}$.

Posterior estimates for $\beta_{p, s}$ and $\gamma_{p, s}$ were obtained using Stan [9]. As a result, three pairs of $\beta$ and $\gamma$ were attributed to each patient. By complementing these values with the average time spent per iteration in each stage, we obtained 9 variables that measure the patient's performance in the GG.

We compared these variables and the MoCA score with respect to predictive power for clinical variables related to automaticity. Specifically, we built classifiers based on either the GG or the MoCA score to predict whether DGI and TUG scores where above or below the median. The classifiers were adjusted using elastic-net regularized logistic regression $[18,68]$. The ROC curves for each pair of explanatory and response variable are presented below.

\section{Results}

Demographic and clinical features of participants are shown in Table 1.

The classifiers in the previous section show that GG variables are at least as good as MoCA for predicting DGI and TUG cost. This relation can be seen in Figure 3. On the one hand, GG variables are better at predicting DGI than MoCA, since the ROC curve for the former is higher than that for the latter: the estimated proportion of correct classifications for DGI with optimum cutoff is $65 \%$ using GG variables and $56 \%$ using MoCA. On the other hand, both GG variables and MoCA have low predictive power for 


\begin{tabular}{llll}
\hline & HY 1 $(\mathrm{n}=23)$ & HY 2 $(\mathrm{n}=31)$ & HY 3 $(\mathrm{n}=20)$ \\
\hline Age (years) & $68.87(9.39)$ & $63.55(8.14)$ & $69.95(8.67)$ \\
Gender (male) & $\mathrm{M}(14)$ & $\mathrm{M}(22)$ & $\mathrm{M}(17)$ \\
Education (years) & $11.30(4.49)$ & $12.90(5.31)$ & $15.40(4.21)$ \\
UPDRS-III & $14.42(7.35)$ & $19.71(7.06)$ & $25.95(11.34)$ \\
MoCA (total) & $24.78(2.70)$ & $24.90(3.43)$ & $24.25(2.77)$ \\
$\quad$ Visuospatial/executive & $3.78(1.13)$ & $3.58(1.09)$ & $3.35(1.46)$ \\
Naming & $2.65(0.65)$ & $2.77(0.43)$ & $2.85(0.37)$ \\
Attention & $5.13(0.97)$ & $5.03(0.91)$ & $4.80(0.95)$ \\
Language & $1.70(0.88)$ & $1.94(0.96)$ & $1.90(0.91)$ \\
Abstraction & $1.87(0.34)$ & $1.84(0.45)$ & $1.80(0.62)$ \\
$\quad$ Memory (delayed recall) & $3.43(1.16)$ & $3.55(1.06)$ & $3.15(0.93)$ \\
Orientation & $5.57(0.59)$ & $5.61(0.80)$ & $5.60(0.60)$ \\
GDS & $4.74(3.00)$ & $4.06(2.53)$ & $5.85(3.23)$ \\
\hline
\end{tabular}

Table 1: Demographic and clinical assessment data of the patients with Parkinson's disease $(\mathrm{n}=74)$. For continuous variables, the mean value is presented and also the standard deviation in parenthesis. For categorical variables, the mode is presented and also the mode's proportion in parenthesis. GDS stands for the geriatric depression scale.

TUG cost: their ROC curves are close to the identity line. When using optimum cutoffs, the estimated proportion of correct classifications for TUG cost using GG variables is $59 \%$ and using MoCA is $57 \%$.

\section{Discussion}

The main finding of this study was that GG has higher power to predict decline in gait performance under complex situations and DT conditions than MoCA. MoCa is a recommended and popular test in PD36, however, as shown elsewhere, it fails to detect gait decline under DT [20]. In face of this, the main purpose of this study was to test whether GG can be used under DT to predict disruption in gait automaticity in early stages of PD evolution. GG allows the use of several strategy trees, deterministic and non-deterministic, which can be tailored to specific experimental protocols.

The GG version adopted in this work was chosen with the purpose of achieving a friendly and free tool able to identify IL deficit regardless of motor control and EL deficits. Thus, the game was designed with 3 phases. The first of them obtains the motor performance baseline, the second evaluates the ability to identify the direction sequence by IL only, and the third evaluates the ability to identify the direction sequence by EL. The motor deficits associated to, e.g., rest tremor or movement slowness, could be isolated as also the possible EL or attentional deficits associated to other cognitive alterations.

IL is a knowledge acquiring process that occurs without conscious awareness of learn- 

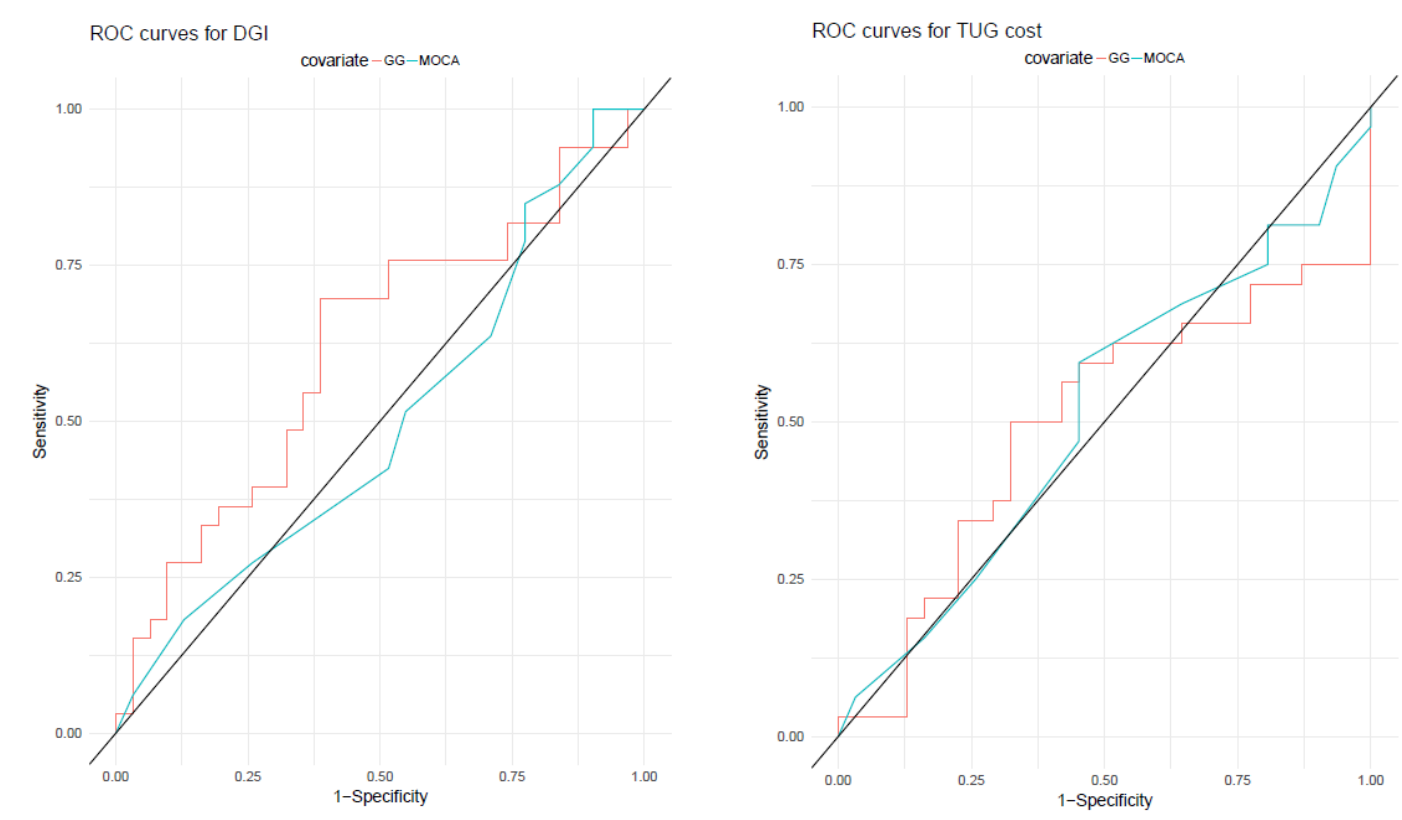

Figure 3: ROC (receiver operator characteristic) curves for predicting DGI (left) and DT-TUG cost (right) using the classifiers adjusted with either GG variables or MoCA score.

ing, whereas EL involves the use of overt strategies. Seminal studies showed that people with amnesia exhibited normal learning of the motor task but had severely impaired EL. In contrast, PPD failed to learn probabilistic classification tasks, despite having intact EL. This double dissociation showed that the limbic-diencephalic regions, damaged in amnesia, and the neostriatum damaged in $\mathrm{PD}$, support separate and parallel learning systems [52,34]. Then, the well-functioning of neostriatum circuits is essential for the gradual, incremental learning of associations that is characteristic of habit learning. In other words, the well-functioning of neostriatum allows the construction of automaticity, i.e., the control of a learned task with minimal attentional demand [64, 48, 24].

Other more recent studies in healthy individuals [16] and PPD have confirmed that neostriatum circuits are associated to implicit sequence learning [62, 54, 23, 26] and probabilistic implicit sequences learning [61, 63, 21].

Therefore, as expected, a tool developed to be sensitive to implicit sequence learning, isolating the influence of motor and EL components, had to be more efficient than a general cognitive test as MoCA to predict the decline in automaticity associate to PD, a disease that has as main pathogenic mechanism dopamine depletion in basal ganglia circuits. Besides, although the comparison of the participants' engagement in doing the GG and MoCA tests was not a purpose of the present study, apparently GG was able to arise more their motivation, imposing a lower level of stress than a paper-and-pencil test as MoCa.

On the other hand, the two gait tests used to assess gait automaticity imposed chal- 
lenging gait conditions for participants where the cognitive resources are more demanded than only walking. Previous studies showed a higher activation in prefrontal cortex during obstacle negotiation in comparison to no obstacle in the gait in young and older adults [39] and PwPD [37]. Even in young healthy individuals the performance level in TUG declined under DT conditions [57]. Taken together, this evidence corroborate that more complex gait conditions demand more cognitive resources and, therefore, are more sensitive to assess gait automaticity than only walking. Besides, the movement sequences tested in TUG and obstacle negotiation tested in DGI are frequently performed in daily life and the performance in these tests is associated with fall risk.

Therefore, it is plausible to hypothesize that GG will be able to predict the level of impairment and fall risk in daily living activity in PPD. To answer this, novel studies should be conducted.

Currently, it has been proposed that early PD could be divided into 3 stages: preclinical in which the neurodegenerative process is started, without evident symptoms or signs of the disease; prodromal, in which symptoms and signs of this disease are present but are insufficient to define a full clinical picture; and clinical, in which the diagnosis is achieved, based on the presence of classical motor signs. Prodromal disease refers to the stage wherein early symptoms or signs of PD neurodegeneration are present, but a clinical diagnosis is not yet possible. Finding new criteria to identify prodromal PD represents a promising challenge for research in PD [38]. In this direction, further studies should be conducted to investigate the ability of GG to identify early decline in gait automaticity in prodromal PD stages.

Finally, it is fundamental that we point some limitations of the present study for result generalization. These are: the small sample size, the absence of a control group and the absence of another test considered as "gold standard" for IL in PD, allowing comparison of results. The deterministic version of game used in our study was able to assess aspects of cognitive tasks performances, making it a tool comparable to MoCA tests for PD. With the development of more strategy trees, including some non-deterministic, and new protocols, it is expected the GG will become a powerful tool in PD screening.

\section{Acknowledgments}

This paper stems from research activity conducted as part of the Research, Innovation and Dissemination Center for Neuromathematics (NeuroMat), funded by the São Paulo Research Foundation-FAPESP (grant 2013/07699-0). MD and YLU are supported by CAPES scholarships. ACR is also recipient of a Brazilian National Council for Scientific and Technological Development-CNPq research productivity grant (\# 306251/2014-0). We thank Antonio Galves for stimulating discussions.

\section{References}

[1] Lisa Alcock, Brook Galna, Jeffrey M Hausdorff, Sue Lord, and Lynn Rochester. Gait \& posture special issue: Gait adaptations in response to obstacle type in fallers 
with parkinson's disease. Gait $\&$ Posture, 61:368-374, 2018.

[2] Marianna Amboni, Paolo Barone, and Jeffrey M Hausdorff. Cognitive contributions to gait and falls: evidence and implications. Movement Disorders, 28(11):1520-1533, 2013.

[3] Katherine Baker, Lynn Rochester, and Alice Nieuwboer. The effect of cues on gait variability - reducing the attentional cost of walking in people with parkinson's disease. Parkinsonism \& Related Disorders, 14(4):314-320, 2008.

[4] Yacov Balash, CH Peretz, G Leibovich, T Herman, JM Hausdorff, and Nir Giladi. Falls in outpatients with parkinson's disease. Journal of Neurology, 252(11):1310$1315,2005$.

[5] Bastiaan R Bloem, Johan Marinus, Quincy Almeida, Lee Dibble, Alice Nieuwboer, Bart Post, Evzen Ruzicka, Christopher Goetz, Glenn Stebbins, Pablo MartinezMartin, et al. Measurement instruments to assess posture, gait, and balance in parkinson's disease: critique and recommendations. Movement Disorders, 31(9): 1342-1355, 2016.

[6] Nicolaas I Bohnen and Klaus Jahn. Imaging: what can it tell us about parkinsonian gait? Movement Disorders, 28(11):1492-1500, 2013.

[7] Stephanie A Bridenbaugh and Reto W Kressig. Motor cognitive dual tasking. Zeitschrift für Gerontologie und Geriatrie, 48(1):15-21, 2015.

[8] Maria Joana D Caetano, Stephen R Lord, Natalie E Allen, Matthew A Brodie, Jooeun Song, Serene S Paul, Colleen G Canning, and Jasmine C Menant. Stepping reaction time and gait adaptability are significantly impaired in people with parkinson's disease: Implications for fall risk. Parkinsonism \& Related Disorders, 47:32-38, 2018.

[9] Bob Carpenter, Andrew Gelman, Matthew D Hoffman, Daniel Lee, Ben Goodrich, Michael Betancourt, Marcus Brubaker, Jiqiang Guo, Peter Li, and Allen Riddell. Stan: A probabilistic programming language. Journal of Statistical Software, 76(1), 2017.

[10] Hui-Ya Chen and Pei-Fang Tang. Factors contributing to single-and dual-task timed "up \& go" test performance in middle-aged and older adults who are active and dwell in the community. Physical Therapy, 96(3):284-292, 2016.

[11] Kelvin L Chou, Melissa M Amick, Jason Brandt, Richard Camicioli, Karen Frei, Darren Gitelman, Jennifer Goldman, John Growdon, Howard I Hurtig, Bonnie Levin, et al. A recommended scale for cognitive screening in clinical trials of parkinson's disease. Movement Disorders, 25(15):2501-2507, 2010.

[12] Elizabeth A Chrischilles, Linda M Rubenstein, Margaret D Voelker, Robert B Wallace, and Robert L Rodnitzky. Linking clinical variables to health-related quality of life in parkinson's disease. Parkinsonism \&5 Related Disorders, 8(3):199-209, 2002. 
[13] David J Clark. Automaticity of walking: functional significance, mechanisms, measurement and rehabilitation strategies. Frontiers in Human Neuroscience, 9:246, 2015.

[14] BA da Silva, CDCM Faria, MP Santos, and A Swarowsky. Assessing timed up and go in parkinson's disease: reliability and validity of timed up and go assessment of biomechanical strategies. Journal of Rehabilitation Medicine, 49(9):723-731, 2017.

[15] Bruno Monte de Castro. Processos Estocásticos Conduzidos por Cadeias com Memória de Alcance Variável e o Jogo do Goleiro. PhD thesis, Universidade de São Paulo, 2016.

[16] Arnaud Destrebecqz, Philippe Peigneux, Steven Laureys, Christian Degueldre, Guy Del Fiore, Joël Aerts, André Luxen, Martial Van Der Linden, Axel Cleeremans, and Pierre Maquet. The neural correlates of implicit and explicit sequence learning: Interacting networks revealed by the process dissociation procedure. Learning 8 Memory, 12(5):480-490, 2005.

[17] Jerome Friedman, Trevor Hastie, and Robert Tibshirani. The Elements of Statistical Learning, volume 1. Springer, 2001.

[18] Jerome Friedman, Trevor Hastie, and Robert Tibshirani. Regularization paths for generalized linear models via coordinate descent. Journal of Statistical Software, 33 (1):1-22, 2010. URL http://www.jstatsoft.org/v33/i01/.

[19] Brook Galna, Anna T Murphy, and Meg E Morris. Obstacle crossing in parkinson's disease: mediolateral sway of the centre of mass during level-ground walking and obstacle crossing. Gait \& Posture, 38(4):790-794, 2013.

[20] Heiko Gaßner, Franz Marxreiter, Simon Steib, Zacharias Kohl, Johannes Schlachetzki, Werner Adler, Bjoern M Eskofier, Klaus Pfeifer, Jürgen Winkler, and Jochen Klucken. Gait and cognition in parkinson's disease: cognitive impairment is inadequately reflected by gait performance during dual task. Frontiers in Neurology, 8: $550,2017$.

[21] Dejan Georgiev, Georg Dirnberger, Leonora Wilkinson, Patricia Limousin, and Marjan Jahanshahi. In parkinson's disease on a probabilistic go/nogo task deep brain stimulation of the subthalamic nucleus only interferes with withholding of the most prepotent responses. Experimental Brain Research, 234(4):1133-1143, 2016.

[22] David J Gill, Arielle Freshman, Jennifer A Blender, and Bernard Ravina. The montreal cognitive assessment as a screening tool for cognitive impairment in parkinson's disease. Movement Disorders, 23(7):1043-1046, 2008.

[23] Eric W Gobel, Kelsey Blomeke, Cindy Zadikoff, Tanya Simuni, Sandra Weintraub, and Paul J Reber. Implicit perceptual-motor skill learning in mild cognitive impairment and parkinson's disease. Neuropsychology, 27(3):314, 2013. 
[24] Ann M Graybiel and Scott T Grafton. The striatum: where skills and habits meet. Cold Spring Harbor Perspectives in Biology, 7(8):a021691, 2015.

[25] Madeleine E Hackney and Gammon M Earhart. Health-related quality of life and alternative forms of exercise in parkinson disease. Parkinsonism $\&$ Related Disorders, 15(9):644-648, 2009.

[26] Heather A Hayes, Nikelle Hunsaker, and Leland E Dibble. Implicit motor sequence learning in individuals with parkinson disease: a meta-analysis. Journal of Parkinson's Disease, 5(3):549-560, 2015.

[27] Taylor R Hendershott, Delphine Zhu, Seoni Llanes, and Kathleen L Poston. Domainspecific accuracy of the montreal cognitive assessment subsections in parkinson's disease. Parkinsonism \& Related Disorders, 38:31-34, 2017.

[28] Margaret M Hoehn, Melvin D Yahr, et al. Parkinsonism: onset, progression, and mortality. Neurology, 50(2):318-318, 1998.

[29] S Hoops, S Nazem, AD Siderowf, JE Duda, SX Xie, MB Stern, and D Weintraub. Validity of the moca and mmse in the detection of mci and dementia in parkinson disease. Neurology, 73(21):1738-1745, 2009.

[30] Sheau-Ling Huang, Ching-Lin Hsieh, Ruey-Meei Wu, Chun-Hwei Tai, Chin-Hsien Lin, and Wen-Shian Lu. Minimal detectable change of the timed "up \& go" test and the dynamic gait index in people with parkinson disease. Physical Therapy, 91(1): 114-121, 2011.

[31] Andrew J Hughes, Susan E Daniel, Linda Kilford, and Andrew J Lees. Accuracy of clinical diagnosis of idiopathic parkinson's disease: a clinico-pathological study of 100 cases. Journal of Neurology, Neurosurgery \& Psychiatry, 55(3):181-184, 1992.

[32] Samyra Keus, Marten Munneke, Mariella Graziano, Jaana Paltamaa, Elisa Pelosin, Josefa Domingos, Susanne Brühlmann, Bhanu Ramaswamy, Jan Prins, Chris Struiksma, et al. Physiotherapy guideline for parkinson's disease. 2013.

[33] Sandra L Kletzel, Juan Manuel Hernandez, Elizabeth F Miskiel, Trudy Mallinson, and Theresa Louise-Bender Pape. Evaluating the performance of the montreal cognitive assessment in early stage parkinson's disease. Parkinsonism 83 Related Disorders, 37:58-64, 2017.

[34] Barbara J Knowlton, Jennifer A Mangels, and Larry R Squire. A neostriatal habit learning system in humans. Science, 273(5280):1399-1402, 1996.

[35] Eiji Kobayashi, Nobuaki Himuro, and Mitsuhiko Takahashi. Clinical utility of the 6min walk test for patients with moderate parkinson's disease. International Journal of Rehabilitation Research, 40(1):66-70, 2017. 
[36] Lillemor Lundin-Olsson, Lars Nyberg, and Yngve Gustafson. Attention, frailty, and falls: the effect of a manual task on basic mobility. Journal of the American Geriatrics Society, 46(6):758-761, 1998.

[37] Inbal Maidan, Freek Nieuwhof, Hagar Bernad-Elazari, Miriam F Reelick, Bas R Bloem, Nir Giladi, Judith E Deutsch, Jeffery M Hausdorff, Jurgen AH Claassen, and Anat Mirelman. The role of the frontal lobe in complex walking among patients with parkinson's disease and healthy older adults: an fnirs study. Neurorehabilitation and Neural Repair, 30(10):963-971, 2016.

[38] Luca Marsili, Giovanni Rizzo, and Carlo Colosimo. Diagnostic criteria for parkinson's disease: From james parkinson to the concept of prodromal disease. Frontiers in Neurology, 9:156, 2018.

[39] Anat Mirelman, Inbal Maidan, Hagar Bernad-Elazari, Shiran Shustack, Nir Giladi, and Jeffrey M Hausdorff. Effects of aging on prefrontal brain activation during challenging walking conditions. Brain and Cognition, 115:41-46, 2017.

[40] Meg E Morris, Jennifer J Watts, Robert Iansek, Damien Jolley, Donald Campbell, Anna T Murphy, and Clarissa L Martin. Quantifying the profile and progression of impairments, activity, participation, and quality of life in people with parkinson disease: protocol for a prospective cohort study. BMC Geriatrics, 9(1):2, 2009.

[41] Susan Morris, Meg E Morris, and Robert Iansek. Reliability of measurements obtained with the timed "up \& go" test in people with parkinson disease. Physical Therapy, 81(2):810-818, 2001.

[42] Ziad S Nasreddine, Natalie A Phillips, Valérie Bédirian, Simon Charbonneau, Victor Whitehead, Isabelle Collin, Jeffrey L Cummings, and Howard Chertkow. The montreal cognitive assessment, moca: a brief screening tool for mild cognitive impairment. Journal of the American Geriatrics Society, 53(4):695-699, 2005.

[43] Frederico Pieruccini-Faria, Jeffery A Jones, and Quincy J Almeida. Motor planning in parkinson's disease patients experiencing freezing of gait: the influence of cognitive load when approaching obstacles. Brain and Cognition, 87:76-85, 2014.

[44] M Plotnik, N Giladi, and JM Hausdorff. Bilateral coordination of gait and parkinson's disease: the effects of dual tasking. Journal of Neurology, Neurosurgery 8 Psychiatry, 80(3):347-350, 2009.

[45] Meir Plotnik, Yaacov Dagan, Tanya Gurevich, Nir Giladi, and Jeffrey M Hausdorff. Effects of cognitive function on gait and dual tasking abilities in patients with parkinson's disease suffering from motor response fluctuations. Experimental Brain Research, 208(2):169-179, 2011.

[46] Moacir Ponti, Patricia Bet, Caroline L Oliveira, and Paula C Castro. Better than counting seconds: Identifying fallers among healthy elderly using fusion of accelerometer features and dual-task timed up and go. PLoS One, 12(4):e0175559, 2017. 
[47] Lynn Rochester, Alice Nieuwboer, Katherine Baker, Victoria Hetherington, A-M Willems, Fabienne Chavret, Gert Kwakkel, Erwin Van Wegen, Inge Lim, and Diana Jones. The attentional cost of external rhythmical cues and their impact on gait in parkinson's disease: effect of cue modality and task complexity. Journal of Neural Transmission, 114(10):1243, 2007.

[48] Shumita Roy, Norman W Park, Eric A Roy, and Quincy J Almeida. Interaction of memory systems during acquisition of tool knowledge and skills in parkinson's disease. Neuropsychologia, 66:55-66, 2015.

[49] Arash Salarian, Fay B Horak, Cris Zampieri, Patricia Carlson-Kuhta, John G Nutt, and Kamiar Aminian. itug, a sensitive and reliable measure of mobility. IEEE Transactions on Neural Systems and Rehabilitation Engineering, 18(3):303-310, 2010.

[50] Robert D Salazar, Xiaolin Ren, Terry D Ellis, Noor Toraif, Olivier J Barthelemy, Sandy Neargarder, and Alice Cronin-Golomb. Dual tasking in parkinson's disease: Cognitive consequences while walking. Neuropsychology, 31(6):613, 2017.

[51] Matej Skorvanek, Jennifer G Goldman, Marjan Jahanshahi, Connie Marras, Irena Rektorova, Ben Schmand, Erik van Duijn, Christopher G Goetz, Daniel Weintraub, Glenn T Stebbins, et al. Reply: Moca for cognitive screening in parkinson's disease: Beware of floor effect. Movement Disorders, 33(3):499-500, 2018.

[52] Larry R Squire and Stuart M Zola. Structure and function of declarative and nondeclarative memory systems. Proceedings of the National Academy of Sciences (USA), 93(24):13515-13522, 1996.

[53] Elizabeth L Stegemöller, Thomas A Buckley, Chris Pitsikoulis, Ernest Barthelemy, Ryan Roemmich, and Chris J Hass. Postural instability and gait impairment during obstacle crossing in parkinson's disease. Archives of Physical Medicine and Rehabilitation, 93(4):703-709, 2012.

[54] Marianne A Stephan, Beat Meier, Sabine Weber Zaugg, and Alain Kaelin-Lang. Motor sequence learning performance in parkinson's disease patients depends on the stage of disease. Brain and Cognition, 75(2):135-140, 2011.

[55] Carolien Strouwen, Esther ALM Molenaar, Samyra HJ Keus, Liesbeth Münks, Elke Heremans, Wim Vandenberghe, Bastiaan R Bloem, and Alice Nieuwboer. Are factors related to dual-task performance in people with parkinson's disease dependent on the type of dual task? Parkinsonism \& Related Disorders, 23:23-30, 2016.

[56] Ruth Tamir, Ruth Dickstein, and Moshe Huberman. Integration of motor imagery and physical practice in group treatment applied to subjects with parkinson's disease. Neurorehabilitation and Neural Repair, 21(1):68-75, 2007.

[57] Kaori Tamura, Morgan Kocher, Liana Finer, Nathan Murata, and Christopher Stickley. Reliability of clinically feasible dual-task tests: Expanded timed get up and go test as a motor task on young healthy individuals. Gait \&3 Posture, 60:22-27, 2018. 
[58] Pei-Fang Tang, Hao-Jan Yang, Ya-Chi Peng, and Hui-Ya Chen. Motor dual-task timed up \& go test better identifies prefrailty individuals than single-task timed up \& go test. Geriatrics \& Gerontology International, 15(2):204-210, 2015.

[59] G Vervoort, E Heremans, A Bengevoord, C Strouwen, E Nackaerts, W Vandenberghe, and A Nieuwboer. Dual-task-related neural connectivity changes in patients with parkinson'disease. Neuroscience, 317:36-46, 2016.

[60] Sally J Vogel, Sarah J Banks, Jeffrey L Cummings, and Justin B Miller. Concordance of the montreal cognitive assessment with standard neuropsychological measures. Alzheimer's \& Dementia: Diagnosis, Assessment $\&$ Disease Monitoring, 1(3):289$294,2015$.

[61] Leonora Wilkinson and Marjan Jahanshahi. The striatum and probabilistic implicit sequence learning. Brain Research, 1137:117-130, 2007.

[62] Leonora Wilkinson, Zunera Khan, and Marjan Jahanshahi. The role of the basal ganglia and its cortical connections in sequence learning: evidence from implicit and explicit sequence learning in parkinson's disease. Neuropsychologia, 47(12):2564$2573,2009$.

[63] Leonora Wilkinson, James T Teo, Ignacio Obeso, John C Rothwell, and Marjan Jahanshahi. The contribution of primary motor cortex is essential for probabilistic implicit sequence learning: evidence from theta burst magnetic stimulation. Journal of Cognitive Neuroscience, 22(3):427-436, 2010.

[64] Tao Wu, Mark Hallett, and Piu Chan. Motor automaticity in parkinson's disease. Neurobiology of Disease, 82:226-234, 2015.

[65] Galit Yogev, Nir Giladi, Chava Peretz, Shmuel Springer, Ely S Simon, and Jeffrey M Hausdorff. Dual tasking, gait rhythmicity, and parkinson's disease: which aspects of gait are attention demanding? European Journal of Neuroscience, 22(5):1248-1256, 2005 .

[66] Galit Yogev, Meir Plotnik, Chava Peretz, Nir Giladi, and Jeffrey M Hausdorff. Gait asymmetry in patients with parkinson's disease and elderly fallers: when does the bilateral coordination of gait require attention? Experimental Brain Research, 177 (3):336-346, 2007.

[67] Galit Yogev-Seligmann, Jeffrey M Hausdorff, and Nir Giladi. The role of executive function and attention in gait. Movement Disorders, 23(3):329-342, 2008.

[68] Hui Zou and Trevor Hastie. Regularization and variable selection via the elastic net. Journal of the Royal Statistical Society: Series B (Statistical Methodology), 67(2): 301-320, 2005. 